\title{
Acute Blood Pressure Response to Different Resistance Programs in Trained Men
}

\author{
Ariani França Conceição, 1,2® Daniell Lima Muniz,, ${ }^{1,2}$ Clarcson Plácido Conceição dos Santos, ${ }^{1,2}$ (『) \\ Ciro Oliveira Queiroz ${ }^{1,2}$ \\ Escola Bahiana de Medicina e Saúde Pública (EBMSP), 'Salvador, BA - Brazil. \\ Grupo de Estudos em Saúde e Performance Humana (GESPH), ${ }^{2}$ Salvador, BA - Brazil
}

\section{Abstract}

Background: Resistance training is used in different exercise programs, with different objectives and different levels of physical fitness. Training-related variables, such as volume, rest time and intensity, can affect the response of blood pressure (BP), but studies on the effect of these variables on BP are still needed.

Objective: To evaluate the acute response of $\mathrm{BP}$ in trained individuals undergoing two different methods of resistance training.

Methods: The sample was divided into three groups: (1) the German volume training (GVT) ( $\mathrm{n}=15)$, which consisted of 10 series of 10 repetitions at $50 \%$ of 1 -repetition maximum (RM) with intervals of 30 seconds; (2) the sarcoplasm stimulating training (SST) $(n=16)$, performed at 8 RM and $85 \%$ of 1 -RM and interval of 10 seconds until failure, followed by removal of $20 \%$ of weight and repetition of the whole series (total of three sets), and the control group (CG) ( $n=15)$ who underwent BP measurements only. The two-way repeated measures ANOVA was used for analysis of variations, and a $\mathrm{p}<0.05$ was considered statistically significant.

Results: In the within-group analysis, a significant lowering of systolic blood pressure (SBP) was found at 10 minutes $(125.4 \pm 10.8 \mathrm{mmHg}, \mathrm{p}=0.045)$ and 20 minutes $(124.5 \pm 8.5 \mathrm{mmHg}, \mathrm{p}=0.044)$ post-training compared with immediately after training. In the between-group comparison, higher SBP values were observed immediately after training in the SST group $(142.1 \pm 28.2, \mathrm{p}=0.048)$ compared with the CG.

Conclusion: High-volume and high-intensity resistance training programs did not cause abnormal changes in blood pressure. (Int J Cardiovasc Sci. 2021; 34(3):294-299)

Keywords: Resistance Training; Exercise; Blood Pressure; Hypertension; Circuit Based Exercise; Exercise; Men; Rehabilitation.

\section{Introduction}

Aerobic and resistance exercises are broadly used for the maintenance and rehabilitation of cardiovascular health, and seen as one of the main non-pharmacological strategies for the prevention and treatment of systemic arterial hypertension (SAH). Resistance training (RT) was long considered a mere auxiliary tool of aerobic exercises for cardiovascular health rehabilitation, particularly for promoting musculoskeletal gain, but today, evidence suggests the efficacy of RT in modesty lowering blood pressure (BP) levels. ${ }^{1-3}$

This phenomenon, known as post-exercise hypotension, has an important role in the control of blood pressure and cardiovascular risks, not only in hypertensive but also in normotensive individuals. ${ }^{4,5}$ The effect of RT on reducing blood pressure levels can prevent the development of SAH in normotensive individuals. ${ }^{4}$ However, there are can affect the response of BP during RT, such as exercise volume, ${ }^{6,7}$ intervals between series ${ }^{8,9}$, training methods ${ }^{10}$ and intensity. ${ }^{11}$

Blood pressure response to exercise intensity and volume is not well established. ${ }^{12}$ Studies have shown that moderate- and high-intensity exercises can lower $\mathrm{BP}$ levels and influence the duration of the hypotensive effect, but not its magnitude. ${ }^{9-11}$ With regard to the volume of exercise, while some reports indicate that the

Mailing Address: Ariani França Conceição

Rua Silveira Martins, 100. Postal Code: 40050-420, Salvador, BA - Brazil.

E-mail: arianiedf@gmail.com 
higher the volume of training, the longer the hypotensive effect, ${ }^{6}$ others have suggested that high- and low-volume training have similar effects on BP. ${ }^{11}$

More advanced methods of RT have been developed over years, such as the German volume training $(\mathrm{GVT})^{13-}$ ${ }^{15}$ and the sarcoplasm stimulating training (SST), ${ }^{16,17}$ but no effect of these programs on BP have been reported so far. Thus, the objective of this study is to assess the acute response of $\mathrm{BP}$ to different methods of $\mathrm{RT}$ in trained individuals.

\section{Methods}

\section{Study design and sample}

This trial included male individuals aged 20-40 years, non-smokers, with at least one year of experience in RT. This was a convenience sample of 46 men, which was divided into three groups: SST $(n=16)$, and GVT $(\mathrm{n}=15)$ and control group (CG) $(\mathrm{n}=15)$.

The exclusion criteria were cardiometabolic diseases, use of medications that may influence the cardiovascular response, musculoskeletal disorders, and individuals who could not undergo the training protocol.

Participants were recruited through social media posts, flyers and telephone calls made to bodybuilding gyms in the city of Salvador, Bahia State, Brazil. Individuals were allocated consecutively in one of the groups, and were informed about the aims of the study, the exercise protocols and training methods. The study was approved by the Research Ethics Committee of Bahian School of Medicine and Public Health (EBMSP) (Certificate of Presentation for Ethical Consideration, CAAE, number 57573516.7.0000.5544). All participants signed an informed consent form.

\section{Anthropometric measures}

On the day before exercise, participants were instructed to refrain from high-intensity exercise, alcohol, caffeinated and energy drinks, and sleep from six to eight hours. On the study day, they were instructed to wear appropriate clothing and eat light meals in the period of two hours before the training.

Body weight and height were measured using a digital scale Welmy® (W200A, Brazil), and subsequently body mass index (BMI) was calculated.

\section{Blood pressure measurements}

An automatic sphygmomanometer - Microlife $®$ (MAM BP3AC1PC, Brazil)- was used. The individuals rested in a supine position for five minutes, to stabilize pressure levels. After five minutes, blood pressure was measured in the pre-training stage, in a supine position, with head slightly tilted, in a quiet environment with a room temperature between $23^{\circ} \mathrm{C}$ and $25^{\circ} \mathrm{C}$ and $\operatorname{dim}$ light. ${ }^{18} \mathrm{BP}$ was then measured immediately after training, and every 10 minutes during the 50 -minute rest period subsequently.

\section{The one-repetition maximum test (1-RM)}

The 1-RM test was used to determine the maximal weight each participant could lift with one repetition. First, the maximal number of repetitions of a given load (percentage of 1-RM) was determined of each participant. For this purpose, participants were first familiarized with the equipment and exercises to be performed. Then, there was a 3-5 minute warm up by performing repetitions of the muscle group to be tested using a light load. After a one-minute interval, participants performed three to five repetitions of a moderate-to-heavy load (60\% to $80 \%$ of predicted 1-RM).

After an interval of two minutes, participants performed repetitions of the load close to the maximum estimated. After performing two-to-three additional repetitions of exercises for the upper limbs, the weight increased by 5 to $10 \%$ of predicted 1-RM. Based on these adjustments, the test would be repeated after an interval of three to five minutes. An attempt was considered valid if the test was performed according to the standardized instructions; if more than three attempts were needed, there was a one-day interval for repetition of the test. ${ }^{19}$

\section{Training Protocol}

\section{German volume training}

Participants performed 10 series of 10 repetitions, with an intensity of $50 \%$ of $1-\mathrm{RM}$, with a 30 -second rest interval between the series. The test was conducted using leg press $45^{\circ}\left(\mathrm{LP} 45^{\circ}\right)$ and bench press (BP). ${ }^{14,20}$

\section{Sarcoplasm stimulating training}

The SST session consisted of eight repetitions (of $85 \%$ of $1-\mathrm{RM}$ ) to failure, i.e., inability to complete a 
full concentric repetition. After a 10-second interval, another set, using the same weight, was performed, so that the participant could make a higher number of repetitions. When a failed attempt occurred, there was an interval of 10 seconds. ${ }^{16}$

Subsequently, $20 \%$ of the weight was removed; participants made another series until failure, followed by one repetition. Then, another $20 \%$ of the weight was removed and the procedure was repeated. ${ }^{16}$ The machines used were $\mathrm{LP} 45^{\circ}$ and BP. ${ }^{20}$

The control group underwent BP measurements and did not participate in any of the exercise protocols.

\section{Statistical analysis}

The statistical package for social sciences (SPSS) software for Windows, version 14.0 was used for the statistical analysis. Continuous variables were described in average and standard deviation, and data normality was tested using the KolmogorovSmirnov test. The independent $\mathrm{t}$-test was used for comparison of 1-RM averages and total weight training between the GVT and SST groups. The oneway ANOVA was used for comparison of mean age and BMI between the groups, and for within-group comparisons of the effect of exercise on systolic BP (SBP) and diastolic BP (DBP) (pre- vs. postintervention). Then, the two-way repeated measures ANOVA was used for comparisons in the pre-and post-intervention periods.

The Bonferroni post-hoc test was used to identify significant differences, at the level of $5 \%$.

\section{Results}

The characteristics of the sample are described in Table 1. No difference was observed in mean age, BMI or 1-RM (BP and $\mathrm{LP} 45^{\circ}$ ) between the groups. However, the total weight of training (LP $45^{\circ}$ and BP) was significantly greater in the GVT than in the SST group ( $p=0.001)$.

Data of SBP and DBP in the pre- and post-training periods are shown in Table 2. In the analyses of SBP, it was observed a significant lowering of SBP in the GVT group at 10 minutes $(\mathrm{p}=0.045)$ and 20 minutes $(\mathrm{p}=0.044)$ compared with immediately after training ( 0 minute). No difference was observed in any of the other time points. No difference was found for DBP in the GVT or SST groups.

Figure 1 shows the comparison of mean SBP and DBP between the three groups in all time points analyzed. Immediately after training, SBP was significantly higher than controls $(p=0.048)$; no other significant differences were found.

\section{Discussion}

The findings of this study showed that the different groups of trained individuals had different acute BP responses to exercises. The within-group comparisons revealed that, in the GVT groups, there was a significant rise in SBP immediately after training compared with 10 minutes and 20 minutes post-exercise.

In a study that compared the effect of different volumes of strength training on BP showed that a higher volume of training caused a more prolonged post-exercise hypotension. ${ }^{6}$ Our results reinforce these

Table 1 - Characteristics of the study groups, composed of trained men undergoing German volume training (GVT, $\mathrm{n}=15)$, the sarcoplasm stimulating training (SST, $n=15)$ and controls ( $=15)$, Salvador, Bahia- Brazil, 2017-2018

\begin{tabular}{|c|c|c|c|c|}
\hline Variables & $\begin{array}{l}C G(n=15) \\
(\text { mean } \pm S D)\end{array}$ & $\begin{array}{l}\text { GVT }(n=15) \\
(\text { mean } \pm S D)\end{array}$ & $\begin{array}{l}\text { SST }(n=16) \\
(\text { mean } \pm S D)\end{array}$ & $\mathrm{p}$ \\
\hline Age (years) & $25.7 \pm 4.9$ & $28 \pm 6.1$ & $26.8 \pm 4.5$ & $0.587^{\#}$ \\
\hline BMI $\left(\mathrm{kg} / \mathrm{m}^{2}\right)$ & $24.7 \pm 3.0$ & $25.6 \pm 2.5$ & $25.6 \pm 2.5$ & $0.503^{\#}$ \\
\hline 1-RM LP $45^{\circ}(\mathrm{kg})$ & - & $312.5 \pm 63.1$ & $315.6 \pm 96.7$ & $0.918^{\$}$ \\
\hline 1-RM BP (kg) & - & $89.1 \pm 19.3$ & $84.7 \pm 18.5$ & $0.522^{\$}$ \\
\hline Full charge LP $45^{\circ}(\mathrm{kg})$ & - & $16503.2 \pm 3569.4$ & $9382.9 \pm 4012.4$ & $0.001^{\$^{*}}$ \\
\hline Full charge BP (kg) & - & $4494.7 \pm 1236.4$ & $2159.0 \pm 1067.1$ & $0.001^{\$^{*}}$ \\
\hline
\end{tabular}


Table 2 - Results of within-group comparisons of mean systolic blood pressure and diastolic blood pressure levels in the pre- vs. post-training in the German volume training (GVT, $n=15)$, sarcoplasm stimulating training (SST, $n=15)$ and control ( $\mathrm{n}=15)$ groups

\begin{tabular}{|c|c|c|c|c|c|c|c|}
\hline & $\begin{array}{c}\text { Pre- } \\
\text { training }\end{array}$ & 0 & 10 & 20 & 30 & 40 & 50 \\
\hline \multicolumn{8}{|l|}{ CG $(n=15)$} \\
\hline SBP mmHg & $125.2 \pm 7.3$ & $124.6 \pm 6.1$ & $126 \pm 5.4$ & $125.1 \pm 7.9$ & $124.7 \pm 7.6$ & $126.5 \pm 6.3$ & $127.9 \pm 12$. \\
\hline DBP mmHg & $69.1 \pm 5.9$ & $67.9 \pm 5.8$ & $69.1 \pm 6.4$ & $68.3 \pm 4.7$ & $71.5 \pm 6$ & $70.8 \pm 7.9$ & $71.7 \pm 7.0$ \\
\hline \multicolumn{8}{|l|}{ GVT (n=15) } \\
\hline SBP mmHg & $127.7 \pm 9.4$ & $137.8 \pm 16.2^{*}$ & $125.4 \pm 10.8^{*}$ & $124.5 \pm 8.5^{*}$ & $126.7 \pm 6.9$ & $123.3 \pm 8.8$ & $124.9 \pm 8.6$ \\
\hline DBP mmHg & $70.9 \pm 9.2$ & $72.5 \pm 13.6$ & $68.5 \pm 8.2$ & $67.2 \pm 5.3$ & $68.6 \pm 7.5$ & $65.3 \pm 9.5$ & $66.5 \pm 6.9$ \\
\hline \multicolumn{8}{|l|}{ SST (n=16) } \\
\hline SBP mmHg & $124.7 \pm 9.2$ & $142.1 \pm 28.2$ & $126.2 \pm 10.5$ & $126 \pm 10.7$ & $122.8 \pm 10.5$ & $123.1 \pm 10.9$ & $122.4 \pm 8.9$ \\
\hline DBP $\mathrm{mmHg}$ & $72.1 \pm 6.8$ & $78.4 \pm 19.7$ & $66.6 \pm 7.4$ & $69.7 \pm 9.2$ & $68.5 \pm 9.7$ & $67.5 \pm 7.9$ & $67.6 \pm 6.3$ \\
\hline
\end{tabular}
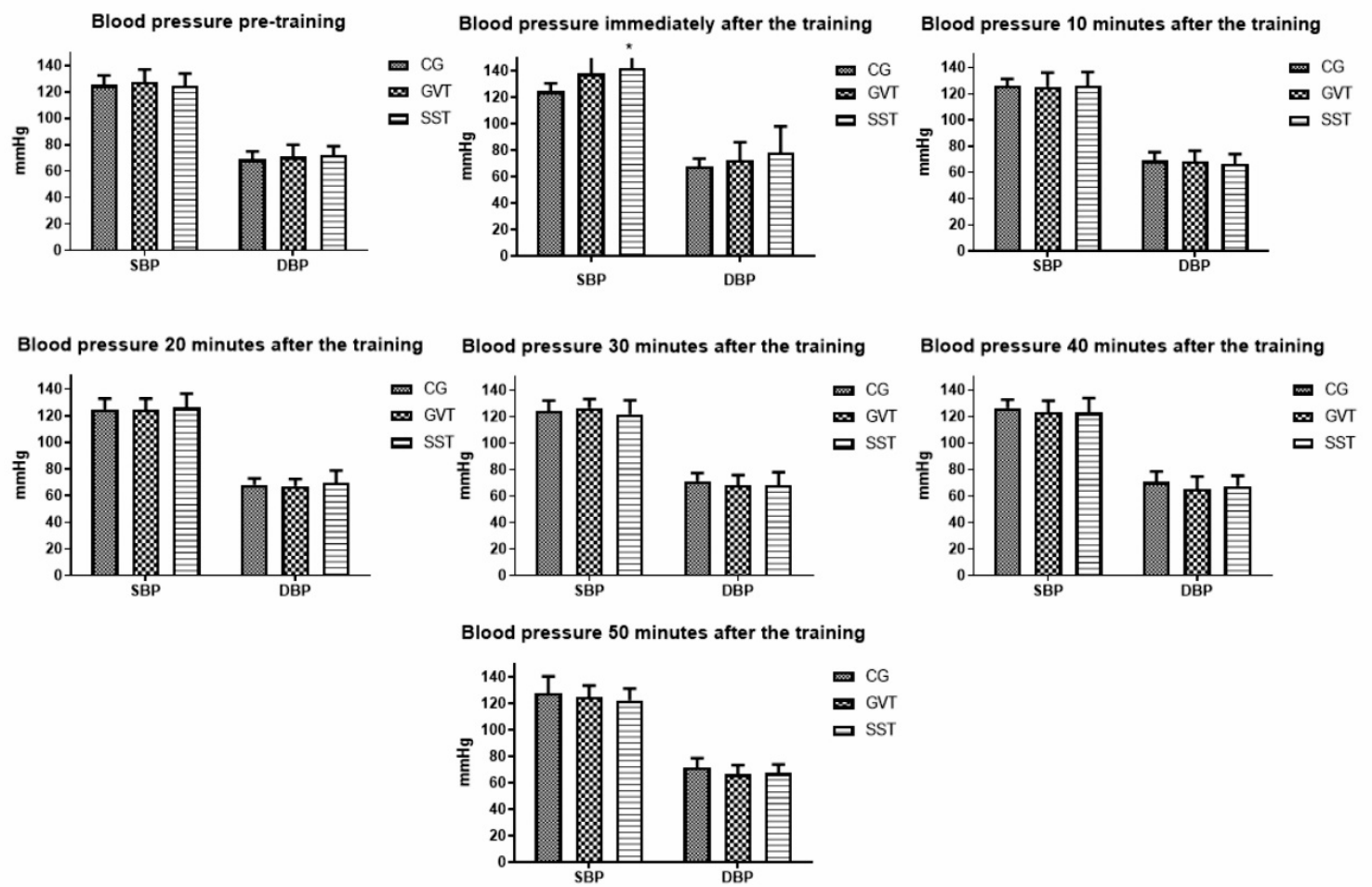

Figure 1 - Comparison of mean systolic blood pressure and mean diastolic blood pressure levels between the German volume training (GVT, $n=15)$, sarcoplasm stimulating training (SST, $n=15)$ and control $(n=15)$ groups CG: control group; GVT: German Volume Training; SST: Sarcoplasm Stimulating Training; mmHg: millimeters of mercury; SBP: systolic blood pressure; DBP: diastolic blood pressure; * $p<0.05$ compared with the control group 
findings, since only GVT, which is an exercise program that involves sets and repetitions with high weights, showed significant hypotension after training. Our data differ from those previously published by Neto et al., ${ }^{11}$ who found similar responses of BP to trainings with different volumes. ${ }^{11}$ However, the total training volume in our study was much higher than the total volume used in their study, which may explain the differences in the results.

Between-group analysis showed significantly higher SBP values in the SST immediately after training compared to the control group. However, despite this rise in BP levels, the higher intensity of the SST method (compared with the GVT) was not able to promote a significant lowering of BP during the post-exercise recovery period. This contrasts with previous studies reporting hypotensive effects of different resistance training intensities. ${ }^{21-23}$

Our data confirm the results of a study ${ }^{24}$ that evaluated the effect of different intensities of exercise, and showed that not only low-intensity exercise ( $40 \%$ of $1-\mathrm{RM})$, but also the ones with high intensity ( $80 \%$ of $1-\mathrm{RM})$ caused systolic arterial hypotension in the post-exercise period. ${ }^{24}$ However, we found a significant lowering of SBP only for the GVT method, which was performed at $50 \%$ of $1-\mathrm{RM}$ (i.e., low intensity).

The highest levels of SBP in the period immediately after training seen in the SST method can be justified by the high intensity and short interval between series ( 10 seconds vs 30 seconds for the GVT method). Thus, there was no time for recovery of pressure levels to start a new series, leading to higher cardiovascular stress. A clinical trial that compared the response of $\mathrm{BP}$ to different rest interval lengths showed that a two-minute interval could be more effective regarding post-exercise hypotension. ${ }^{25}$ Although our trainings had shorter duration, the significant lowering of BP after the GVT may be explained by a longer rest interval between the series. ${ }^{25}$

Another hypothesis for the significantly rise in SBP levels immediately after the SST was the occurrence of fatigue in most of the repetitions. This is in line with the study by Polito and Farinatti, ${ }^{26}$ who noticed that the highest BP elevations occurred when fatigue was reached after all repetitions. ${ }^{26}$ Higher values of SBP was observed in the SST compared with the GVT group; in fact, the SST proposes that individuals perform the series until fatigue, which differs from the GVT, where fatigue occurred mostly in the last repetitions.

Regarding DBP, no significant effect by any of the methods was found. DBP levels remained below the pre-training levels until the end of the analysis period. This result contrasts with the study showing that exercise with lower intensity would lower DBP for a short period, while exercises with higher intensity would not change acute DBP responses. ${ }^{27}$

One of the limitations in our study refers to the measurement of BP in the supine position. This is supported by the study ${ }^{28}$ that evaluated the influence of posture on the recovery of $\mathrm{BP}$ and heart rate after resistance training in normotensive individuals. The study found greater hypotensive responses in the sitting position during recovery than in the supine position. ${ }^{28}$ However, more studies are needed to support this hypothesis.

Our study group was composed of young male trained, normotensive adults. More studies are needed including female, older and hypertensive individuals. It is worth highlighting that studies on GVT and SST are still scarce in the literature, and that this is the first study to investigate the acute effects of these trainings on BP.

\section{Conclusion}

There was a significant lowering of SBP at 10 and 20 minutes after the GVT compared with the immediate post-training. The between-group analysis showed significantly higher SPB levels in the immediate posttraining in the SST group compared with control group. High-volume RT of high-intensity-RT did not cause abnormal changes in BP.

\section{Potential Conflict of Interest}

No potential conflict of interest relevant to this article was reported.

\section{Sources of Funding}

There were no external funding sources for this study.

\section{Study Association}

This study is not associated with any thesis or dissertation work.

\section{Ethics approval and consent to participate}

This study was approved by the Ethics Committee of the Escola Bahiana de Medicina e Saúde Human aunder the protocol number CAAE: 57573516.7.0000.5544. All the procedures in this study were in accordance with the 1975 
Helsinki Declaration, updated in 2013. Informed consent was obtained from all participants included in the study.

\section{Author contributions}

Conception and design of the research: Queiroz CO, Santos CPD, Conceição AF. Acquisition of data:
Conceição AF, Queiroz CO, Santos CPD, Muniz DLC. Analysis and interpretation of the data: Conceição AF, Queiroz CO, Muniz, DLC. Statistical analysis: Conceição AF, Queiroz CO. Writing of the manuscript: Conceição AF, Queiroz CO. Critical revision of the manuscript for intellectual content: Queiroz CO.

\section{References}

1. Précoma DB, Oliveira GMM, Simão AF, Dutra OP, Coelho OR, Izar MCO, et al. Atualização da Diretriz de Prevenção Cardiovascular da Sociedade Brasileira de Cardiologia - 2019. Arq Bras Cardiol. 2019;113(4):787-891.

2. Herrod PJJ, Doleman B, Blackwell JEM, O'Boyle F, Williams JP, Lund JN, et al. Exercise and other nonpharmacological strategies to reduce blood pressure in older adults: a systematic review and meta-analysis. J Am Soc Hypertens. 2018;12(4):248-67.

3. Sharman JE, Gerche AL, Coombes JS. State of the art exercise and cardiovascular risk in patients with hypertension. Am J Hypertens. 2015; 28(2):147-58.

4. Pescatello, LS; Franklin, BA; Fagard, R; Farquhar, WB; Kelley, GA; Ray C. American College of Sports Medicine. Exercise and hypertension. Med Sci Sport Exerc. 2004; 36(3):533-53.

5. Pickering TG, Hall JE, Appel LJ, Falkner BE, Graves J, Hill MN, et al. Recommendations for blood pressure measurement in humans and experimental animals: Part 1: Blood pressure measurement in humans - A statement for professionals from the subcommittee of professional and public education of the American Heart Association Council on High Blod Pressure Research. Circulation. 2005;111(5):697-716.

6. Figueiredo T, Rhea MR, Peterson M, Miranda H, Bentes CM, Machado de Ribeiro dos Reis V, et al. Influence of number of sets on blood pressure and heart rate variability after a strength training session. J Strength Cond Res. 2015;29(6):1556-63.

7. Castinheiras-Neto AG, Costa-Filho IR, Farinatti PTV. Cardiovascular Responses to Resistance Exercise are Affected by Workload and Intervals between Sets. Arq Bras Cardiol. 2010;95(4):493-501.

8. Veloso J, Polito MD, Riera T, Celes R, Vidal JC, Bottaro M. Effects of rest interval between exercise sets on blood pressure after resistance exercises. Arq Bras Cardiol. 2010;94(4)512-8.

9. Rodriguez D, Nakazato K, Fleck S, Pontes Jr LF, Charro MA, Alegretti G, et al. Strength training methods does not affect post- exercise hypotension and heart rate variability. J Exerc Physiol online. 2017;20(5):36-51.

10. Neto VGC, Figueiredo T, Simões AD, Bezerra MG, Barguti STP, Bentes $\mathrm{CM}$, et al. La influencia de la intensidad de la carga sobre la presión arterial después de una sesión de entrenamiento de fuerza. Apunt Med l'Esport. 2017;52(193):23-8.

11. Neto VGC, Fonseca EA, Damasco CAA, Oliveira RS, Bentes CM, Miranda H. [Hipotensão pós-treinamento de força realizado em sessões com diferentes intensidades]. Rev Bras Prescrição e Fisiol do Exerc. 2017;11(71):912-9.

12. Rivera CE, Jiménez MJ, Rojas SW, Herrera SA. Acute effects of exercise on blood pressure: a meta-analytic investigation. Arq Bras Cardiol. 2016;106(5):422-33.

13. Baker D. German Volume Training: an alternative method of high volume-load training for stimulating muscle growth. Perform Train J. 2009;8(1):10-3

14. Amirthalingam T, Mavros Y, Wilson GC, Clarke JL, Mitchell L, Hackett DA. Effects of a modified German Volume Training program on muscular hypertrophy and strength. J Strength Cond Res. 2017;31(11):3109-19.

15. Gambassi BB, Queiroz C, Muniz D, Conceição A, Santos CE, Galvão V, et al. Acute effect of German Volume Training method on autonomic cardiac control of apparently healthy young. J Exerc Physiol online. 2019;22(2):49-57.

16. Prestes J, Foschini D, Marchetti P, Charro MA, Tibana R. Prescrição e Periodização do treinamento de força em academias. $2^{\mathrm{a}}$ ed. Barueri: Manole; 2016.

17. Almeida FN, Lopes CR, Machado R, Oenning L, Crisp AH, Manuel $\mathrm{N}$, et al. Acute effects of the new method sarcoplasma stimulating training versus traditional resistance training on total training volume, lactate and muscle thickness. Front Physiol. 2019;10:1-7.

18. Malachias M, Souza W, Plavnik F, Rodrigues C, Brandão A, Neves $\mathrm{M}$, et al. $7^{\mathrm{TH}}$ Brazilian Guideline of Arterial Hypertension. Arq Bras Cardiol. 2016;107(supl 3):1-83.

19. Adams GM. Exercise Physiology Laboratory Manual. Boston; 1998.

20. Maior AS, Varallo ÂT, Matoso AG, Edmundo DA, Oliveira MM, Minari VA. [Resposta da força muscular em homens com a utilização de duas metodologias para o teste de 1RM]. Rev Bras Cineantropometria Desempenho Hum. 2007;9(2):177-82.

21. Casonatto J, Goessler KF, Cornelissen VA, Cardoso JR, Polito MD. The blood pressure-lowering effect of a single bout of resistance exercise: a systematic review and meta-analysis of randomised controlled trials. Eur J Prev Cardiol. 2016;23(16):1700-14.

22. Bentes CM, Costa PB, Neto GR, Costa G V, Salles BF de, Miranda HL, et al. Hypotensive effects and performance responses between different resistance training intensities and exercise orders in apparently health women.Clin Physiol Funct Imaging. 2014;35(5):185-90.

23. Figueiredo T, Willardson JM, Miranda H, Bentes CM, Reis VM, Simão $R$. Influence of load intensity on postexercise hypotension and heart rate variability after a strength training session. J Strength Cond Res. 2015;29(10):2941-8

24. Rezk CC, Marrache RCB, Tinucci T, Mion D, Forjaz CLM. Postresistance exercise hypotension, hemodynamics, and heart rate variability: influence of exercise intensity. Eur J Appl Physiol. 2006;98(1):105-12.

25. Figueiredo T, Willardson J, Mranda H, Bentes CM, Reis VM, Salles BF, et al. Influence of rest interval length between sets on blood pressure and heart rate variability after a strength training session performed by prehypertensive men. J Strength Cond Res. 2016;30(7):1813-24.

26. Polito M, Farinatti PTV. [Respostas de frequência cardíaca, pressão arterial e duplo produto ao exercício contra resistência - uma revisão da literatura]. Rev Port Ciências do Desporto. 2003;3(1):79-91.

27. Polito MD, Simão R,Senna WG, Farinatti PTV. Hypotensive effects of resistance exercises performed at different intensities and same work volumes. Rev Bras Med Esporte. 2003;9(2):69-73.

28. Farinatti PTV, Nakamura FY, Polito MD. Influence of recovery posture on blood pressure and heart rate after resistance exercises in normotensive subjects. J Strength Cond Res. 2009;23(9):2487-92. 